\title{
UNKNOTTED SOLID TORI AND GENUS ONE WHITEHEAD MANIFOLDS
}

\author{
EDWARD M. BROWN
}

\begin{abstract}
In this paper we study contractible open 3-manifolds which are monotone unions of solid tori and which embed in a compact 3-manifold. We show that the tori are unknotted in later tori. We then study pairs of unknotted solid tori, and prove a unique prime decomposition theorem. This is applied to the open 3-manifolds above to get an essentially unique prime decomposition. A number of examples in the literature are analyzed, and some new examples are constructed.
\end{abstract}

\section{INTRODUCTION}

This paper studies those open subsets of compact 3-manifolds which are contractible and have genus 1 at $\infty$ (see $\S 1$ for definitions). If such a subset is irreducible then it is a union of a monotone sequence of solid tori. These have been called genus one Whitehead manifolds $[11,13]$ in honor of J. H. C. Whitehead who exhibited the first one in 1935 in $[18,19]$. Whitehead's example was described again by Bing in [1]. If $W$ denotes Whitehead's example then Glimm and Shapiro showed $W \times \mathbb{R}^{1}$ is homeomorphic to $\mathbb{R}^{4}$, and Glimm [4] showed that $W \times W$ is homeomorphic to $\mathbb{R}^{6}$. McMillan and Zeeman showed that 'homeomorphic' can be replaced by 'combinatorially equivalent' in [12]. McMillan [10] extended Whitehead's construction to get an uncountable family of topologically distinct genus one Whitehead manifolds embedded in the 3 -sphere $S^{3}$. In the same paper McMillan studied an example of a genus one Whitehead manifold which Bing had conjectured would not embed in $S^{3}$. Later Kister and McMillan returned to Bing's example in [8] and showed that it, and uncountably many similar examples, do not embed in $S^{3}$. In [5], Haken showed that these examples do not embed in any compact 3-manifold. Poénaru [15] proved that any solid torus in a simply connected 3-manifold is a stage in the construction of a genus one Whitehead manifold. This author [2] showed that each member of a class of 3-manifolds, which includes all genus $n$ Whitehead manifolds, is topologically determined by its proper homotopy type. McMillan and Thickstun showed in [11] that a genus $n$ Whitehead manifold which embeds in a compact 3-manifold having no homotopy sphere factors, also embeds in $S^{3}$. In the same paper they also point out by a cardinality argument that some genus one Whitehead manifold is not the universal cover of any compact

Received by the editors August 8, 1990 .

1980 Mathematics Subject Classification (1985 Revision). Primary 57N10, 57M40.

Key words and phrases. Whitehead manifold, open 3-manifold, contractible manifold. 
3-manifold, but their argument does not produce any specific example. In [13], Myers showed that a genus one Whitehead manifold is not a nontrivial cover of any 3-manifold, in particular, it is not the universal cover of any compact 3-manifold.

Our results in this paper are the following. We first make the simple observation that a contractible open set in a compact 3-manifold which has genus 1 at $\infty$ is the connected sum of an irreducible such set with a homotopy 3sphere. We then observe in Lemma 4.3 that when it is irreducible such a set is a union of solid tori; that is, it is a genus one Whitehead manifold (a genus one Whitehead manifold is irreducible). Suppose that $O=\bigcup\left\{V_{n}\right\}$ is a genus one Whitehead manifold in a compact 3-manifold that is given as the union of the solid tori $V_{n}$. Using arguments of Haken's, we show in Theorem 4.4 that for a sufficiently large $n$, the pair $\left(V_{n+1}, V_{n}\right)$ of solid tori has an embedding in $S^{3}$ with both solid tori unknotted (we call this an unknotted pair). Work of Schubert [16] shows that if $(A, B)$ is a nontrivial unknotted pair, then there is a decomposition $B=E_{0} \subset E_{1} \subset \cdots \subset E_{n}=A$ into a sequence of solid tori $\left\{E_{k}\right\}$ so that every pair $\left(E_{k+1}, E_{k}\right)$ is unknotted and prime. The heart of the paper is Theorem 3.1 which shows that the decomposition is unique up to isotopy $\operatorname{rel} B$. This leads to the main Theorem, 4.6, that a genus one Whitehead manifold which embeds in a compact 3-manifold has an essentially unique prime decomposition. (The word essentially here refers to the fact that one can change a sequence $\left\{V_{n}\right\}$ by drawing an arbitrary solid torus $V$ inside $V_{1}$ and renumbering to make it the first term.) Myers proved Theorem 4.6 in the special case that the sequence $\left\{V_{n}\right\}$ is what he calls excellent; that is, any essential annulus in $V_{n}^{c} \cap V_{n+1}$ is boundary parallel, and $V_{n}$ is contractible in $V_{n+1}$ (then $\left(V_{n+1}, V_{n}\right)$ is prime). As Myers points out, Whitehead's example is excellent, but McMillan's examples are not. We show by example that there are uncountably many excellent genus one Whitehead manifolds. By example we also show the assumption that the genus one Whitehead manifold embeds in a compact 3-manifold is necessary for Theorem 4.6. This is because a pair $(A, B)$ of solid tori can have distinct prime decompositions if it is not unknotted. We point out that McMillan's examples show that if one only assumes that $\left(V_{n+1}, V_{n}\right)$ is prime among contractible pairs (and not among all pairs) then there are prime decompositions that have no terms in common. We also show an example of a genus one Whitehead manifold with a decomposition $\left\{V_{n}\right\}$ such that $\left(V_{n+1}, V_{n}\right)$ is prime and unknotted for every $n$, but for infinitely many $n$ 's, $\left(V_{n+2}, V_{n}\right)$ is a knotted pair. This manifold embeds in no compact 3-manifold. We show that a genus one Whitehead manifold which embeds in a compact 3-manifold also embeds in $S^{3}$.

The paper is organized as follows. In $\S 1$ we give the basic definitions. In $\S 2$ we show that certain pairs of solid tori, called twisted pairs, are prime. In $\S 3$ we state and prove the unique prime decomposition theorem for unknotted pairs of solid tori. In $\S 4$ we prove the theorems about open 3-manifolds, and give most of the examples.

\section{Definitions}

We work entirely in the P.L. category. Much of our notation is taken from $[6,7]$. The symbols $\simeq$ and $\sim$ mean (P.L.) homeomorphic to and homotopic to 
respectively. A solid torus is a space $A$ with $S^{1} \times D^{2} \simeq A$ (unit circle $\times$ unit disk). If $h: S^{1} \times D^{2} \simeq A$ then $h\left(S^{1} \times\{p\}\right), p \in \operatorname{int}\left(D^{2}\right)$, is a core of $A$, while $h\left(\{p\} \times D^{2}\right), p \in S^{1}$, is a meridian disk, and $h\left(\{p\} \times \partial D^{2}\right)$ is a meridian of $A$. Any two meridian disks are isotopic in $A$, and cores are isotopic $\operatorname{rel} \partial A$. Any simple closed curve in $\partial A$ which is essential in $\partial A$ but null-homotopic in $A$ is a meridian.

Suppose $A \subset S^{3}$ is a solid torus. By $A^{c}$ we mean the closure of $S^{3}-A$; more generally, $A^{c}$ is the closure of $M-A$ where $M$ is the largest 3-manifold so far mentioned which contains $A$. A longitude of $A$ is a simple closed curve which is essential in $\partial A$, but null-homologous in $A^{c} . A$ is unknotted provided a core of $A$ is the unknot in $S^{3}$; equivalently, $A^{c}$ is a solid torus, which is true iff a longitude of $A$ bounds a disk in $A^{c}$. Thus, if $A$ is unknotted, a longitude of $A$ is a meridian of $A^{c}$.

$(A, B)$ is a pair of solid tori if $A$ and $B$ are solid tori and $B$ is contained in the interior of $A$ (we write $B \subset A$ ). A pair $(A, B)$ of solid tori is unknotted if there exists an embedding $h: A \rightarrow S^{3}$ with $h(A)$ and $h(B)$ both unknotted. In this case we will frequently identify $(A, B)$ with $(h(A), h(B))$ and think of $(A, B)$ as embedded in $S^{3}$. Associated to a pair $(A, B)$ of solid tori there are two integers, the winding number, $W(A, B)$, and the wrapping number, $N(A, B)$. The winding number is the nonnegative coefficient of a core of $B$ in the fundamental group $\pi_{1}(A)$. This is zero iff $B$ is contractible in $A$. The wrapping number $N(A, B)$ is the minimum number of points in which any core of $B$ meets a meridian disk of $A$ (cf. [16]). These numbers depend only on the isotopy class of $B$ in $A$. The following is an easy remark.

Remark 1.1. Suppose $(A, B)$ is an unknotted pair. Then $N(A, B)=0$ iff $B$ is contained in a cell in $A$ and $N(A, B)=1$ iff a core of $B$ is also a core of $A$. In each case there is, up to isotopy of $B$ in $A$, exactly one such pair $(A, B)$.

An unknotted pair $(A, B)$ is called a trivial pair if $N(A, B)<2$.

Remark 1.2. Suppoe $A \supset B \supset C$ are solid tori. Then

$$
W(A, C)=W(A, B) * W(B, C) \text { and } N(A, C)=N(A, B) * N(B, C) .
$$

Proof. The first equation is obvious, the second is in [16].

A nontrivial unknotted pair $(A, C)$ is said to factor if there exists a solid torus $B$ with $A \supset B \supset C$, so that $(A, B)$ and $(B, C)$ are both nontrivial unknotted pairs. Otherwise we say that $(A, C)$ is a prime pair. By Remark 1.2 , if $N(A, C)$ is a prime integer, then the unknotted pair $(A, C)$ is a prime pair. In particular, any nontrivial pair $(A, C)$ has a finite prime factorization.

The next theorem is mainly due to Schubert [16] and shows that the pairs $(A, B)$ and $(B, C)$ above are automatically unknotted.

Theorem 1.3. Let $(A, C)$ be an unknotted pair with $N(A, C)>0$. Suppose $T \subset A$ is a torus which separates $\partial A$ from $\partial C$. Then $T$ bounds a solid torus $B$ in $A$ so that $(A, B)$ and $(B, C)$ are both unknotted.

Proof. Embed $(A, C)$ in $S^{3}$ so that both are unknotted, and let $B$ be the closure of the component of $S^{3}-T$ which contains $C$. Suppose, first, that $B$ is a solid torus. Choose a meridian disk $D$ of $C^{c}$ so that $D$ meets $T$ in general position and in a minimal number of components (which are simple 
closed curves). Any component of $D \cap T$ which is inessential on $T$ bounds disks on both $D$ and $T$. But such components can be removed by the usual disk swapping arguments, so all components of $D \cap T$ are essential on $T$. Let $\gamma$ be a component of $D \cap T$ which bounds an innermost disk $D_{1}$ on $D$. If $D_{1} \subset B$ then $D_{1}$ is a meridian disk of $B$ since $\gamma$ is essential on $T$. But a meridian disk of $B$ must meet $\partial D$, for otherwise $C$ is contained in a cell in $B$ (and hence in $A$ ). We conclude that $D_{1} \subset B^{c}$, so $B$ is unknotted in $S^{3}$.

Finally, suppose $B^{c}$ is a solid torus. Then making the same argument with respect to $\left(C^{c}, A^{c}\right)$ we conclude that $B^{c}$, and hence $B$, is unknotted.

It follows from the above that an unknotted nontrivial pair $(A, C)$ is prime iff every essential torus in $A \cap C^{c}$ is boundary parallel $\left(A \cap C^{c}\right.$ is sometimes called simple).

If $O$ is an open connected 3-manifold then $O=\bigcup\left\{C_{n}\right\}$ where each $C_{n}$ is a compact 3-manifold, and $C_{n}$ is contained in the interior of $C_{n+1}$. The sequence $\left\{C_{n}\right\}$ has been called an exhausting sequence, or a defining sequence for $O$. If we can choose $\left\{C_{n}\right\}$ with the genus of $\partial C_{n}$ bounded, then we call the smallest bound for the genus of $\partial C_{n}$ the genus of $O$ at $\infty$. Notice that if the genus of $O$ at $\infty$ is $g$, we can choose $\left\{C_{n}\right\}$ so that no $C_{n}$ is contained in a compact submanifold $C$ of $O$ with the genus of $\partial C$ less than $g$.

\section{TWISTED PAIRS}

An unknotted pair $(A, B)$ is called a twisted pair if a core of $B$ is parallel to a curve on $\partial A$. This means there is an annulus $\alpha$ embedded in $A$ so that $\alpha \cap \partial A$ is one boundary component of $\alpha$ and the other boundary component of $\alpha$ is a core of $B$. The main result of this section is that a twisted nontrivial pair is prime.

In what follows we shall often speak of a meridian-longitude pair for a solid torus $A$ embedded in $S^{3}$. This means a pair $(m, l)$ consisting of a meridian $m$ and a longitude $l$ for $A$ which intersect in one point. We give $A$ the orientation induced from $S^{3}$ and $\partial A$ the orientation induced from $A$. We consider the curves $m$ and $l$ to be oriented so that $(m, l)$, in that order, induces the positive orientation on $\partial A$. Note that $(-m,-l)$ is also a meridian-longitude pair, but $(-m, l)$ and $(m,-l)$ are not.

Lemma 2.1. Let $(A, B)$ be a twisted nontrivial pair considered as embedded in $S^{3}$. Let $b$ be a curve on $\partial A$ that is parallel to a core of $B$. Then for an appropriately oriented meridian-longitude pair $(m, l)$ of $A$ we have $b \sim$ $k * l \pm m$, where $k=W(A, B)=N(A, B)$.

Proof. The curve $b$ is nonsingular on $\partial A$, and so $b \sim k * l+j * m$ for relatively prime integers $k \geq 0$ and $j$. Neither $k$ nor $j$ is zero since $(A, B)$ is nontrivial, and $k \neq 1$ for the same reason. If neither $k$ nor $j$ were \pm 1 , then $b$ would be a torus knot (see [3]) but a core of $B$ is not parallel to a knot in $S^{3}$. Thus $j= \pm 1$ as desired.

Clearly $W(A, B)=k$, but a curve such as $b$ meets some meridian disk in $k$ points. It follows that there is a meridian disk of $A$ which meets a core of $B$ in $k$ points so $N(A, B)=k$.

Lemma 2.2. Let $(A, B)$ be a nontrivial pair, unknotted in $S^{3}$, and let $(m, l)$, $\left(m^{\prime}, l^{\prime}\right)$ be meridian-longitude pairs for $A$ and $B$ respectively. Then the pair 
$(A, B)$ is a twisted pair iff there is a properly embedded annulus $\alpha \subset A \cap B^{c}$ with $\alpha \cap \partial A \sim k * l \pm m$, and $\alpha \cap \partial B \sim k * m^{\prime} \pm l^{\prime}$.

Proof. If $\alpha$ exists then $\alpha \cap \partial B$ is parallel in $B$ to a core of $B$, so one implication is clear.

Suppose $(A, B)$ is a twisted pair and $\gamma$ is an annulus with one boundary a core of $B$ and the other the curve $k * l \pm m$ on $\partial A$. Suppose further that $\gamma$ is chosen to have general position with respect to $\partial B$, and so that $\gamma \cap \partial B$ has a minimal number of components. A component of $\gamma \cap \partial B$ is inessential on $\partial B$ if and only if it is inessential on $\gamma$. For $\pi_{1}(\gamma) \rightarrow \pi_{1}(A)$ is a monomorphism, so an essential curve on $\gamma$ is essential in $A$, and hence on $\partial B$. If a component of $\gamma \cap \partial B$ is inessential in $\gamma$, we can select one that bounds an innermost disk $D$. Suppose $\partial D$ is essential on $\partial B$. If $D \subset B$, then $D$ is a meridian disk of $B$ which misses a core of $B$. If $D \subset B^{c}$ then $B$ is contained in a cell in $A$, but both are false. Any component of $\gamma \cap \partial B$ which is inessential on both $\partial B$ and $\gamma$ can be removed by disk swapping, so none exist.

The set $\gamma \cap \partial B$ has only one component since any two would be parallel on $\partial B$ contradicting the minimal number. Let $\alpha=\gamma \cap B^{c}$, a subannulus of $\gamma$. Now $\alpha \cap \partial A=\gamma \cap \partial A$ which says that one boundary component of $\alpha$ is as desired. The other boundary, $\alpha \cap \partial B$, is parallel in $B$ to a core of $B$. It follows that $\alpha \cap \partial B \sim k^{\prime} * m^{\prime} \pm l^{\prime}$. If $(m, l)$ is a meridian-longitude pair for $A$, then $(l, m)$ is a meridian-longitude pair for $A^{c}$. Thus $\alpha \cap \partial A$ is parallel to a core of $A^{c}$, which shows that $\left(B^{c}, A^{c}\right)$ is also a twisted pair. Finally, $k$ and $k^{\prime}$ are both the linking number of a core of $B$ with a core of $A^{c}$, and so are equal.

Corollary 2.3. A pair $(A, B)$ is a nontrivial twisted pair iff $\left(B^{c}, A^{c}\right)$ is a nontrivial twisted pair.

Lemma 2.4. Suppose $A \supset B \supset C$ is a nontrivial factoring of the twisted pair $(A, C)$. Then $(A, B)$ and $(B, C)$ are also twisted pairs.

Proof. Let $\alpha$ be an annulus joining a core of $C$ to a curve on $\partial A$. Suppose $\alpha$ is chosen to have general position with respect to $\partial B$, and so that $\alpha \cap \partial B$ has a minimal number of components. Since $\pi_{1}(\alpha) \rightarrow \pi_{1}(A)$ is a monomorphism, if a component of $\alpha \cap \partial B$ is inessential on $\partial B$, then it is inessential on $\alpha$ as well. On the other hand, if there are any components of $\alpha \cap \partial B$ which are inessential on $\alpha$, we may choose an innermost one $b$ bounding a disk $D \subset \alpha$. If $b$ is essential on $\partial B$ and $D \subset B$, then $D$ is a meridian disk of $B$ which misses a core of $C$. But $(B, C)$ was assumed nontrivial. Next, if $D \subset B^{c}$, then a regular neighborhood of $B \cup D$ is a cell in $A$ containing $B$. We conclude that $b$ is inessential on $\partial B$. But then disk swapping eliminates $b$. Thus all components of $\alpha \cap \partial B$ are essential on both $\alpha$ and on $\partial B$. Since $\partial B$ separates $\partial A$ from $\partial C, \alpha \cap \partial B \neq \varnothing$. It follows that there is a subannulus of $\alpha$, contained in $B$, bounded by a core of $C$ and a curve on $\partial B$. We conclude that $(B, C)$ is a twisted pair.

If $(A, C)$ is a twisted pair then by Corollary $2.3,\left(C^{c}, A^{c}\right)$ is a twisted pair and $C^{c} \supset B^{c} \supset A^{c}$ is a nontrivial factorization. As above we conclude that $\left(B^{c}, A^{c}\right)$ is a twisted pair, and, again by Corollary 2.3 , that $(A, B)$ is also a twisted pair.

Theorem 2.5. If $(A, C)$ is a nontrivial twisted pair, then $(A, C)$ is a prime pair. 
Proof. Suppose to the contrary that $A \supset B \supset C$ is a nontrivial factorization with $W(A, B)=k$ and $W(B, C)=r$. Let $\alpha$ be an annulus in $A \cap C^{c}$ with $\alpha \cap \partial A \sim k * r * l \pm m$ and $\alpha \cap \partial C \sim k * r * m^{\prime \prime} \pm l^{\prime \prime}$, where $(m, l)$ and $\left(m^{\prime \prime}, l^{\prime \prime}\right)$ are meridian-longitude pairs for $A$ and $C$ respectively (Lemma 2.2). We assume $\alpha$ is chosen so that it has general position and minimal intersection with $\partial B$. As usual, $\alpha$ meets $\partial B$ in curves essential on both $\alpha$ and $\partial B$. Since these curves are parallel on $\partial B$ there is only one of them, call it $b$. Now $\alpha$ can be extended slightly inside $C$ to a core, so by Lemma $2.1 b \sim r * l^{\prime} \pm m^{\prime}$, where $\left(m^{\prime}, l^{\prime}\right)$ is a meridian-longitude pair for $B$. Similarly, $\alpha$ can be extended a little inside $A^{c}$ to a core of $A^{c}$. Again by Lemma 2.1 applied to $\left(B^{c}, A^{c}\right)$, $b \sim k * m^{\prime} \pm l^{\prime}$. It follows that $k=r=1$, which contradicts nontriviality.

\section{UNIQUE PRIME DECOMPOSITION}

By a prime decomposition of a nontrivial unknotted pair $(A, B)$ we mean a sequence $B=E_{0} \subset E_{1} \subset \cdots \subset E_{k}=A$, where each pair $\left(E_{t+1}, E_{t}\right)$ is prime. As noted in the introduction, a prime decomposition exists for any nontrivial pair $(A, B)$, our main theorem is uniqueness.

Theorem 3.1. Let $B=E_{0} \subset E_{1} \subset \cdots \subset E_{k}=A$ and $B=E_{0}^{\prime} \subset E_{1}^{\prime} \subset \cdots \subset E_{l}^{\prime}=$ $A$ be two prime decompositions of the nontrivial pair $(A, B)$, unknotted in $S^{3}$. Then $k=l$ and there is an isotopy of $A$, fixing points of $\partial A \cup B$, of the identity to a homeomorphism which carries $E_{r}$ to $E_{r}^{\prime}$ for all $r$.

Proof. If the theorem is false, select a counterexample so that $k$ and then $l$ are as small as possible. Let $T_{r}=\partial E_{r}$ and $T_{r}^{\prime}=\partial E_{r}^{\prime}$. By an isotopy fixing $B \cup \partial A$ move the $E_{r}$ so that $T_{r}$ meets $T_{t}^{\prime}$ in general position for all $r$ and $t$, and so that the number of path components of $T_{r} \cap T_{t}^{\prime}$, \# $\pi_{0}\left(T_{r} \cap T_{t}^{\prime}\right)$, is as small as possible for all $r$ and $t$.

(1) The inclusion maps induce monomorphisms $\pi_{1}\left(T_{r}\right) \rightarrow \pi_{1}\left(A \cap B^{c}\right)$, and $\pi_{1}\left(T_{r}^{\prime}\right) \rightarrow \pi_{1}\left(A \cap B^{c}\right)$. Otherwise, $B$ is contained in a cell in $A$.

(2) Components of $T_{r} \cap T_{t}^{\prime}$ are essential on both $T_{r}$ and $T_{t}^{\prime}$. Any inessential components bound disks on both $T_{r}$ and on $T_{t}^{\prime}$ by (1). Then using disk swapping we can decrease \# $\pi_{0}\left(T_{r} \cap T_{t}^{\prime}\right)$.

(3) Components of $T_{r} \cap \bigcup_{t} T_{t}^{\prime}$ are parallel on $T_{r}$, and components of $T_{t}^{\prime} \cap \bigcup_{r} T_{r}$ are parallel on $T_{t}^{\prime}$, since they are pairwise disjoint essential curves.

Suppose $T_{m} \cap T_{n}^{\prime} \neq \varnothing$. Choose a meridian disk $D_{m}$ of $E_{m}$ so that $D_{m}$ meets $T_{n}^{\prime}$ in general position and $\# \pi_{0}\left(D_{m} \cap T_{n}^{\prime}\right)$ is as small as possible. The rest of the proof breaks into two cases.

Case I. For some $m, n$, and $D_{m}$, some component of $D_{m} \cap T_{n}^{\prime}$ is a simple closed curve. (Case II will be that every such component is an arc.)

(4) Every simple closed curve component of $D_{m} \cap T_{n}^{\prime}$ is essential on $T_{n}^{\prime}$. Otherwise the usual disk swapping argument will decrease $\# \pi_{0}\left(D_{m} \cap T_{n}^{\prime}\right)$.

(5) The solid tori $E_{m}$ and $E_{n}^{\prime}$ have a common meridian disk. Let $c$ be a component of $D_{m} \cap T_{n}^{\prime}$ which bounds a disk $D^{\prime}$ in $D_{m}$ that contains no further points of $T_{n}^{\prime}$ (i.e., $c$ is innermost on $D_{m}$ ). Since $c$ is essential on $T_{n}^{\prime}$ by (4), and $E_{n}^{\prime}$ is not contained in a cell in $A, D^{\prime} \subset E_{n}^{\prime}$. Thus $D^{\prime}$ is a meridian disk of $E_{n}^{\prime}$. There is an annulus component $\alpha$ of $T_{n}^{\prime} \cap E_{m}$ which contains $c$, and $c$ is essential on $\alpha$. Let $\alpha^{\prime}$ be one of the two subannuli into which $c$ cuts $\alpha$. Then $\alpha^{\prime} \cup D^{\prime}$ is a meridian disk of $E_{m}$. Having the same boundary, but slightly to one side, is a common meridian disk of $E_{m}$ and $E_{n}^{\prime}$. 
(6) If $E_{m}$ and $E_{n}^{\prime}$ have a common meridian disk $D$, then there is an isotopy keeping $\partial A \cup B$ pointwise fixed and moving $E_{m}$ onto $E_{n}^{\prime}$. If $E_{m}=E_{n}^{\prime}=A$, or $E_{m}=E_{n}^{\prime}=B$ then there is nothing to prove. We assume $0<m<k$ and $0<$ $n<1$. Now $c=\partial D$ is a component of $T_{m} \cap T_{n}^{\prime}$. By (3) components of $E_{m} \cap T_{n}^{\prime}$ are annuli bounded by meridians of both $E_{m}$ and $E_{n}^{\prime}$ as are components of $T_{m} \cap E_{n}^{\prime}$. It follows that the component $F$ of $E_{m} \cap E_{n}^{\prime}$ containing $B$ is bounded by a torus separating $\partial B$ from $\partial A$. Thus $F$ is an unknotted solid torus. Since $D \subset E_{m} \cap E_{n}^{\prime}$ and $\partial D$ is essential on $\partial F, D$ is a meridian disk of $F$. Shrinking $F$ inside itself a little we have $N\left(E_{m}, F\right)=N\left(E_{n}^{\prime}, F\right)=1$, so there are isotopies of the desired kind moving $E_{m}$ to $F$ and $F$ to $E_{n}^{\prime}$.

(7) In Case I, $E_{m}=E_{n}^{\prime}=A$ or $E_{m}=E_{n}^{\prime}=B$. Otherwise (6) gives a contradiction to the choice of $k$ and $l$.

This finishes Case I.

Case II. If $0<m<k$ and $T_{n}^{\prime} \cap T_{m} \neq \varnothing$, then every component of $D_{m} \cap T_{n}^{\prime}$ is an arc with endpoints in $\partial D_{m}$. Note that $T_{n}^{\prime} \cap D_{m} \neq \varnothing$, for otherwise $E_{n}^{\prime}$ and $E_{m}$ have a common meridian disk and (6) gives a contradiction.

(8) $T_{1} \cap T_{1}^{\prime} \neq \varnothing$. Otherwise, either $T_{1}^{\prime} \subset E_{1}$ or $T_{1} \subset E_{1}^{\prime}$. In the first case we have $E_{0} \subset E_{1}^{\prime} \subset E_{1}$. Since $\left(E_{1}, E_{0}\right)$ is prime $N\left(E_{1}, E_{1}^{\prime}\right)=1$, so $E_{1} \cap E_{1}^{\prime c} \simeq T_{1} \times[0,1]$. Then the isotopy of the required kind can be performed in a small neighborhood of $E_{1} \cap E_{1}^{\prime c}$ moving $E_{1}$ onto $E_{1}^{\prime}$. This contradicts the choice of $k$ and $l$. The proof is similar in the case $T_{1} \subset E_{1}^{\prime}$.

The components of $T_{1}^{\prime} \cap\left(\bigcup_{r} T_{r}\right)$ cut $T_{1}^{\prime}$ into annuli denoted $\left\{\alpha_{1, t}^{\prime}\right\}$. Since we are in Case II, $T_{1}^{\prime} \cap D_{1}$ is a family of arcs each being a component of the intersection of an annulus $\alpha_{1, t}^{\prime}$ with $D_{1}$. The endpoints of these arcs are in both $\partial D_{1}$ and $\partial \alpha_{1, t}^{\prime}$ and they cut $D_{1}$ into a family of subdisks. One of these subdisks is called outermost if its boundary consists of a single arc on $\partial D_{1}$ and a single arc of $\alpha_{1, t}^{\prime} \cap D_{1}$. Clearly there are outermost subdisks.

(9) If $\alpha_{1, t}^{\prime} \cap D_{1} \neq \varnothing$ then each component spans $\alpha_{1, t}^{\prime}$ (i.e., meets both boundary components of $\left.\alpha_{1, t}^{\prime}\right)$. Suppose some component of $\alpha_{1, t}^{\prime} \cap D_{1}$ has both endpoints on the same component of $\partial \alpha_{1, t}^{\prime}$. Then it cuts off a disk $D^{\prime}$ from $\alpha_{1, t}^{\prime}$ and we may choose $D^{\prime}$ so that $D^{\prime} \cap D_{1} \subset \partial D_{1}$ (i.e., $D^{\prime}$ is outermost on $\alpha_{1, t}^{\prime}$ ). The arc $D^{\prime} \cap D_{1}$ cuts $D_{1}$ into two subdisks. We can swap $D^{\prime}$ with either of these subdisks to change $D_{1}$ to another embedded disk in $E_{1}$ since $D^{\prime}$ is outermost. One of these disks will be a meridian disk of $E_{1}$ which, after a small displacement, decreases $\# \pi_{0}\left(D_{1} \cap T_{1}^{\prime}\right)$.

(10) Suppose $D$ is an outermost subdisk of $D_{1}$ and $\alpha^{\prime}=\alpha_{1, t}^{\prime}$ is the annulus on $T_{1}^{\prime}$ that cuts off $D$. Then there is an annulus $\alpha \subset T_{1}$ so that $\partial \alpha=\partial \alpha^{\prime}$ and $\alpha \cap T_{1}^{\prime}=\partial \alpha$. The boundary $\partial \alpha^{\prime}$ separates $T_{1}$ into two annuli, let $\alpha$ be the one that contains the arc $a=\partial D \cap T_{1}$. Since $D$ is outermost, $a \cap T_{1}^{\prime}=\partial a=a \cap \alpha^{\prime}$. But $a$ spans $\alpha^{\prime}$ by (9) and so spans $\alpha$. A spanning arc of $\alpha$ meets any essential curves on $\alpha$, thus $\alpha \cap T_{1}^{\prime}=\partial \alpha$.

Fix a pair $\alpha, \alpha^{\prime}$ as in (10).

(11) The arcs $\alpha \cap \partial D$ and $\alpha^{\prime} \cap D$ are spanning arcs of $\alpha$ and $\alpha^{\prime}$ respectively. See (10).

(12) If $D$ is outermost on $D_{1}$ then $D \cap E_{0} \neq \varnothing$. Otherwise, pushing through the disk $D$, we can change $\alpha$ from an annulus to a disk, and then remove it completely. This decreases $\# \pi_{0}\left(T_{1} \cap T_{1}^{\prime}\right)$. 
Let $T=\alpha \cup \alpha^{\prime}$, a torus, and let $F$ be the closure of the component of $S^{3}-T$ containing $D$ (and also $E_{0}$ by (12)). By Theorem 1.3, $F$ is an unknotted solid torus. Since $E_{0} \subset F \subset E_{1}$, either $N\left(F, E_{0}\right)=1$ or $N\left(E_{1}, F\right)=1$ and $N\left(E_{1}^{\prime}, F\right)=1$.

(13) $N\left(F, E_{0}\right)=1$. Otherwise shrink $F$ slightly inside itself using a regular neighborhood of the boundary. Then $N\left(E_{1}, F\right)=N\left(E_{1}^{\prime}, F\right)=1$. But the same contradiction as in (8) shows this cannot happen.

(14) The pairs $\left(E_{1}, E_{0}\right)$ and $\left(E_{1}^{\prime}, E_{0}\right)$ are twisted pairs. The disk $D$ is a meridian disk of $F$, so a component $b$ of $\partial \alpha$ is parallel to a core of $F$. By (13) a core of $E_{0}$ is a core of $F$, and is thus parallel in $F$ to $b$. Since $b \subset T_{1} \cap T_{1}^{\prime}$, both pairs above are twisted pairs.

(15) $T_{1}^{\prime} \cap T_{2}=\varnothing$. Otherwise one of the annuli $\alpha_{1, t}^{\prime}$ lies in $E_{2} \cap E_{1}^{c}$ and has one boundary on $T_{1}$ and the other on $T_{2}$. But the component of $\partial \alpha_{1, t}^{\prime}$ lying on $T_{1}$ is parallel on $T_{1}$ to $b$ (see (14)). Then a core of $E_{0}$ is parallel to a curve on $T_{2}$ and the pair $\left(E_{2}, E_{0}\right)$ is a twisted pair. This contradicts Theorem 2.5 which says that a twisted pair is prime.

(16) $k=l=2$, and the prime decompositions of our counterexample are $E_{0} \subset E_{1} \subset E_{2}$, and $E_{0} \subset E_{1}^{\prime} \subset E_{2}$. By (15) $E_{1}^{\prime} \subset E_{2}$ and is part of a prime decomposition of $\left(E_{2}, E_{0}\right)$. Thus $k=2$ and $E_{0} \subset E_{1}^{\prime} \subset \cdots \subset E_{1}^{\prime}=E_{2}$. The argument repeated with the roles of $E_{m}$ and $E_{m}^{\prime}$ interchanged shows that $l=2$ as well.

We can apply the above arguments to the prime decompositions $E_{2}^{c} \subset E_{1}^{c} \subset$ $E_{0}^{c}$, and $E_{2}^{c} \subset E_{1}^{\prime c} \subset E_{0}^{c}$. We conclude that

(17) The pair $\left(E_{1}^{c}, E_{2}^{c}\right)$ is a twisted pair with a core of $E_{2}^{c}$ parallel to the same $b$ as a core of $E_{0}$. Both cores are parallel to a component of $T_{1} \cap T_{1}^{\prime}$.

Now let $(m, l)$ be a meridian-longitude pair for $E_{1}$. Since $\left(E_{1}, E_{0}\right)$ is a twisted pair by (14), Lemma 2.1 says that $b \sim k * l \pm m$, where $k=N\left(E_{1}, E_{0}\right)$. Since $(l, m)$ is a meridian-longitude pair for $E_{1}^{c}$, (17) says that $b \sim k^{\prime} * m \pm l$. Thus $k=k^{\prime}=1$ which contradicts the fact that the pair $\left(E_{1}, E_{0}\right)$ is nontrivial. This contradiction finishes the proof.

\section{Contractible open 3-MANifolds}

In this section $O$ (or $O^{\prime}$, etc.) will always be a contractible open set of genus 1 at $\infty$ in a compact 3-manifold $M$ (or $M^{\prime}$, etc.). The sequence $\left\{V_{n}\right\}$ will be an exhausting (or defining) sequence for $O$ such that $\partial V_{n}$ has genus 1, no $V_{n}$ is contained in a compact submanifold of $O$ with boundary of genus $O$, and $\partial V_{n}$ is not parallel to $\partial V_{n+1}$. It follows from the loop theorem of [17] that $\pi_{1}\left(\partial V_{n}\right) \rightarrow \pi_{1}\left(V_{0}^{c} \cap V_{n}\right)$ and $\pi_{1}\left(\partial V_{n}\right) \rightarrow \pi_{1}\left(V_{n}^{c} \cap O\right)$ are both monomorphisms (here $V_{n}^{c}$ is with respect to $\left.M\right)$. Then by [14] or [9], $\pi_{1}\left(\partial V_{n}\right) \rightarrow \pi_{1}\left(V_{0}^{c} \cap O\right)$ is also a monomorphism.

Theorem 4.1. If $O$ (as above) is not irreducible then there is a 2-sphere $\Omega \subset O$ which separates $M$ and gives a decomposition $O \simeq O^{\prime} \# S$ where $S$ is a nontrivial homotopy 3-sphere and $O^{\prime}$ is an irreducible contractible open 3-manifold of genus 1 at $\infty$. Also $M \simeq M^{\prime} \# S$ where $M^{\prime}$ is compact and $O^{\prime} \subset M^{\prime}$.

Proof. If $O$ is not irreducible there is a 2-sphere $\Omega_{1} \subset O$ which does not bound a ball in $O$. Since $O$ is contractible, $\Omega_{1}$ separates $O$. Since $O$ has only one end (being contractible), $\Omega_{1}=\partial A_{1}$ for some compact $A_{1} \subset O$. Then $\pi_{1}\left(A_{1}\right) \rightarrow \pi_{1}(M)$ is a monomorphism and factors through $\pi_{1}(O)$. If 
$S_{1}$ is $A_{1}$ capped off with a cell, then $S_{1}$ is a nontrivial homotopy sphere and $O \simeq O_{1}^{\prime} \# S_{1}$, with $M \simeq M_{1}^{\prime} \# S_{1}$ and $O_{1} \subset M_{1}$. If $O_{1}$ is not irreducible we can find a 2-sphere $\Omega_{2}$ in $O_{1}$ bounding a homotopy cell $A_{2}$ containing $\Omega_{1}$. Again let $S_{2}$ be $A_{2}$ capped, so $M \simeq M_{2}^{\prime} \# S_{1} \# S_{2}$, and $O \simeq O_{2}^{\prime} \# S_{1} \# S_{2}$. Since $M$ has a finite prime decomposition this must stop after some number $k$ of steps and $S=S_{1} \# \ldots \# S_{k}, \Omega=\partial A_{k}^{\prime}, O^{\prime}=O_{k}^{\prime}$, and $M^{\prime}=M_{k}^{\prime}$ are as desired.

Lemma 4.2. If $O$ (as above) is irreducible then each $V_{n}$ is a solid torus.

Proof. Since $\pi_{1}\left(\partial V_{n}\right) \rightarrow \pi_{1}\left(V_{n}^{c} \cap O\right)$ is a monomorphism, $\pi_{1}\left(\partial V_{n}\right) \rightarrow \pi_{1}\left(V_{n}\right)$ has a nontrivial kernel. The loop theorem provides a properly embedded disk $D$ in $V_{n}$ with $\partial D$ essential in $\partial V_{n}$. Since $O$ is contractible it is orientable and so is $V_{n}$. Since $O$ is irreducible, cutting $V_{n}$ over $D$ gives a cell, so $V_{n}$ is a solid torus.

Lemma 4.3. For sufficiently large $n, \pi_{1}\left(\partial V_{n}\right) \rightarrow \pi_{1}\left(V_{n}^{c}\right)$ has a nontrivial kernel. Proof. By the Haken finiteness theorem [5], [6], or [7] the compact 3-manifold $V_{0}^{c}$ does not contain an infinite number of nonparallel, incompressible tori. It follows that all but a finite number of the $\partial V_{n}$ are compressible in $V_{0}^{c}$. Since $\partial V_{n}$ is incompressible in $V_{n} \cap V_{0}^{c}, \partial V_{n}$ is compressible in $V_{n}^{c}$ if it is compressible in $V_{0}^{c}$.

From now on, in addition to our other assumptions, we assume that $O$ is irreducible, that every $V_{n}$ is a solid torus, and that $\partial V_{n}$ is compressible in $V_{n}^{c}$ for every $n$.

In [1], Bing conjectured that if $V_{n}$ has a local knot in $V_{n+1}$ (i.e., meets some cell in a single knotted tube) then $O$ does not embed in $S^{3}$. In [8], Kister and McMillan proved Bing's conjecture. Haken [5] extended this to show that $O$ does not even embed in a compact 3-manifold.

By a longitude of a solid torus $A$ we mean a curve which is carried to a longitude by some unknotted embedding of $A$ in $S^{3}$. By a longitude pair for an unknotted pair $(A, B)$ we mean a pair of curves on $(\partial A, \partial B)$ which are carried to longitudes by an unknotted embedding of the pair $(A, B)$ in $S^{3}$.

Theorem 4.4. For $O$ and $\left\{V_{n}\right\}$ as above, there is a longitude $l_{n}$ of $V_{n}$ for each $n$ so that for every $n$ and $k,\left(V_{n+k}, V_{n}\right)$ is an unknotted pair, and $\left(l_{n+k}, l_{n}\right)$ is a longitude pair.

Proof. Let $K_{n}=V_{n} \cup U\left(D_{n}\right)$ where $D_{n}$ is a compressing disk for $\partial V_{n}$ in $V_{n}^{c}$. Here $U\left(D_{n}\right)$ is a regular neighborhood of $D_{n}$ in $V_{n}^{c}$ so that $U\left(D_{n}\right) \cap \partial V_{n}$ is a regular neighborhood of $\partial D_{n}$ in $\partial V_{n}$ (i.e., an annulus). I claim that $K_{n}$ is a cell. The argument below follows Haken's argument [5, p. 68].

To see that $K_{n}$ is a cell, choose a meridian loop $m_{n}$ on $\partial V_{n}$ which meets $\partial D_{n}$ in general position and in a minimal number $r$ of points. We show that $r=1$. Let $l$ be a simple loop on $\partial V_{n}$ which meets $m_{n}$ transversally in a single point. Then $l$ generates $H_{1}\left(V_{n}\right)$ and $l, m_{n}$ together generate $H_{1}\left(\partial V_{n}\right)$. As $r$ is minimal, with appropriately chosen orientations, $\partial D_{n}$ is homologous to $r * l+s * m_{n}$ on $\partial V_{n}$. Moreover $\partial D_{n}$ is a simple closed curve, so $r$ and $s$ are relatively prime. It follows that $\pi_{1}\left(K_{n}\right)$ is isomorphic to $Z / r Z$ (the integers $\bmod r)$ generated by $l$. Since $U\left(D_{n}\right) \cap \partial V_{n}$ is an annulus, $\partial K_{n}$ is a 2 -sphere. Then $\pi_{1}(M)$ is the free product of $\pi_{1}\left(K_{n}\right)$ with $\pi_{1}\left(K_{n}^{c}\right)$, so $\pi_{1}\left(K_{n}\right) \rightarrow \pi_{1}(M)$ is a monomorphism. But the generator $l$ of $\pi_{1}\left(K_{n}\right)$ lies in $V_{n}$ and hence in $O$, so it is null homotopic in $M$. It follows that $r=1$, and that $K_{n}$ is a cell. 
Let $l_{n}=\partial D_{n}$ for each $n$. Since $l_{n}$ is inessential in $V_{n}^{c}$, it is inessential in $V_{n}^{c} \cap K_{n+k}$. Thus the pair $\left(V_{n+k}, V_{n}\right)$ is unknotted in the cell $K_{n+k}$, and $\left(l_{n+k}, l_{n}\right)$ is a pair of longitudes.

In [10] McMillan gave a construction that showed:

Theorem 4.5. Suppose $\left\{\left(A_{n}, B_{n}\right)\right\}$ is a sequence of unknotted pairs. There is an open subset $O \subset S^{3}$, and an exhausting sequence of solid tori $\left\{V_{n}\right\}$ for $O$, so that $\left(V_{n+1}, V_{n}\right) \simeq\left(A_{n}, B_{n}\right)$ for every $n$. If there are arbitrarily large $n$ for which $B_{n}$ is contractible in $A_{n}$, then $O$ is contractible.

By Theorem 4.4, $O$ has an exhausting sequence $\left\{V_{n}\right\}$ of solid tori so that $\left(V_{n+1}, V_{n}\right)$ is unknotted for every $n$. If $\left(V_{n+1}, V_{n}\right)$ is prime for every $n$, we call the sequence a prime sequence. As we have noted, every such $O$ has a prime sequence. The next theorem says that essentially there is only one prime sequence for $O$.

Theorem 4.6. Suppose $O$ and $O^{\prime}$ are as above, with prime sequences $\left\{V_{n}\right\}$ and $\left\{V_{n}^{\prime}\right\}$. If $O \simeq O^{\prime}$ then there are integers $k$ and $l$, and a homeomorphism $h: O \rightarrow O^{\prime}$ so that $h\left(V_{n+k}\right)=V_{n+l}^{\prime}$ for every $n$.

Proof. As noted before, $\left\{V_{n}\right\}$ can be extended by adding arbitrary terms at the beginning so the integers $k$ and $l$ cannot be eliminated.

Let $h^{\prime}: O \rightarrow O^{\prime}$ be a homeomorphism, and choose integers $k$ and $m$ so that $V_{1}^{\prime} \subset h^{\prime}\left(V_{k}\right) \subset V_{m}^{\prime}$. By Theorem 3.1 applied to the pair $\left(V_{m+1}^{\prime}, V_{0}^{\prime}\right)$, there is an isotopy of $O^{\prime}$, fixed on $V_{0}^{\prime} \cup V_{m+1}^{\prime c}$ carrying $h^{\prime}\left(V_{k}\right)$ to $V_{l}^{\prime}$ for some $l$. We let $h_{0}$ be the composition of $h^{\prime}$ with the end of this isotopy.

Suppose, for some $n \geq 0$, we have constructed a homeomorphism $h_{n}$ of $O$ to $O^{\prime}$ with $h_{n}\left(V_{k+t}\right)=V_{l+t}^{\prime}$ for all $0 \leq t \leq n$. Choose $m$ with $V_{l+n}^{\prime} \subset$ $h_{n}\left(V_{k+n+1}\right) \subset V_{m}^{\prime}$. Apply Theorem 3.1 to $\left(V_{m+1}^{\prime}, V_{l+n}^{\prime}\right)$ to get an isotopy, fixed on $V_{l+n}^{\prime} \cup V_{m+1}^{\prime c}$, and moving $h_{n}\left(V_{k+n+1}\right)$ onto $V_{l+n+1}^{\prime}$. We let $h_{n+1}$ be the composition of $h_{n}$ with the end of this isotopy. The desired homeomorphism is the limit of the sequence $\left\{h_{n}\right\}$.

We would now like to show that every $O$ (as above) can be embedded in $S^{3}$. This was proved in [11] in the case that $M$ is irreducible and orientable.

Lemma 4.7. If $\left(V_{n+1}, V_{n}\right)$ is an unknotted pair with longitude pair $\left(l_{n+1}, l_{n}\right)$, then any unknotted embedding $f_{n}: V_{n} \rightarrow S^{3}$ with $f_{n}\left(l_{n}\right)$ a longitude, extends to an unknotted embedding $f_{n+1}: V_{n+1} \rightarrow S^{3}$ with $f_{n+1}\left(l_{n+1}\right)$ a longitude.

Proof. Let $h$ be an unknotted embedding of $\left(V_{n+1}, V_{n}\right)$ in $S^{3}$ which carries the pair $\left(l_{n+1}, l_{n}\right)$ to longitudes. We may even assume that $f_{n}\left(V_{n}\right)=h\left(V_{n}\right)$ and $f_{n}\left(l_{n}\right)=h\left(l_{n}\right)$ by composing $h$ with a homeomorphism of $S^{3}$. Now $f_{n} \circ h^{-1}: h\left(V_{n}\right) \rightarrow h\left(V_{n}\right)$ and maps a longitude to itself. It follows that $f_{n} \circ h^{-1}$ extends to a homeomorphism $H: S^{3} \rightarrow S^{3}$. We claim that $f_{n+1}=H \circ h$ is as desired.

Clearly, $f_{n+1}\left|V_{n}=H \circ h\right| V_{n}=f_{n} \circ h^{-1} \circ h \mid V_{n}=f_{n}$. Since $h\left(l_{n+1}\right)$ is a longitude of $h\left(V_{n+1}\right)$, and $H$ is defined on all of $S^{3}$, it follows that $f_{n+1}\left(l_{n+1}\right)$ is a longitude of $f_{n+1}\left(V_{n+1}\right)$.

Corollary 4.8. With notation as in Theorem 4.4, there is an embedding $f: O \rightarrow$ $S^{3}$ with $f\left(l_{n}\right)$ a longitude of $f\left(V_{n}\right)$ for every $n$.

Consider now the examples of genus one Whitehead manifolds constructed by McMillan [10, Theorem 1]. They are all of this form: Select a sequence 
$\left\{n_{k}\right\}$ of integers, all greater than 1 . For each $k$ let $A_{k} \supset B_{k} \supset C_{k}$ be unknotted solid tori in $S^{3}$ so that $\left(A_{k}, B_{k}\right)$ is a twisted pair with $N\left(A_{k}, B_{k}\right)=n_{k}$ and $\left(B_{k}, C_{k}\right)$ is the Whitehead pair. Then $\left\{V_{k}\right\}$ with $\left(V_{2 k+2}, V_{2 k+1}\right) \simeq\left(A_{k}, B_{k}\right)$ and $\left(V_{2 k+1}, V_{2 k}\right) \simeq\left(B_{k}, C_{k}\right)$ is a prime sequence for a contractible open set in $S^{3}$. By Theorem 4.6, two sequences $\left\{n_{k}\right\}$ and $\left\{n_{k}^{\prime}\right\}$ give nonhomeomorphic open sets unless they are cofinally equal. Notice that the pairs $\left(V_{2 k+3}, V_{2 k+1}\right)$ and $\left(V_{2 k+2}, V_{2 k}\right)$ are prime among contractible pairs. This gives decompositions $\left\{V_{2 k+1}\right\}$ and $\left\{V_{2 k}\right\}$ which are prime sequences among contractible pairs for the same open set, but have no terms in common. Thus Theorem 4.6 is false if "prime" is replaced with "prime among contractible pairs".

Here is a list of infinitely many prime torus pairs (all of which have wrapping number 2) from which many more examples can be constructed (see Figure 1). In an unknotted solid torus $A \subset S^{3}$ select a cell in the form $D^{2} \times[0,1]$. In the cell select an arc $a \subset \operatorname{int}\left(D^{2}\right)$ and a ribbon going from $a \times 0$ to $a \times 1$ which ties a knot $k$, and which makes $n 1 / 2$-twists (say c.c.w. if $n>0$, and c.w. if $n<0)$. The edge of the ribbon is a pair of wires in $D^{2} \times[0,1]$. Connect the ends of the wires in $D^{2} \times 1$ with an arc running around $A$, and similarly for the ends in $D^{2} \times 0$. A regular neighborhood gives an unknotted solid torus $B$ in $A$ with wrapping number 2. The winding number is 0 if $n$ is even, and 2 if $n$ is odd. By examining the inverse image of $B$ in the 2-fold cyclic cover of $A$, one can see that the homeomorphism type of $(A, B)$ changes if $k$ or $n$ is changed. The assumption that the pair $(A, B)$ is unknotted cannot be dropped from Theorem 3.1. We can get examples of this using the fact that knot multiplication is commutative. Let $(A, C)$ be the pair so that $N(A, C)=1$, and so that $C$ ties two local prime knots, say a figure eight and a trefoil, as it circles $A$ (see Figure 2). There are two prime decompositions of $(A, C): A \supset B \supset C$ where

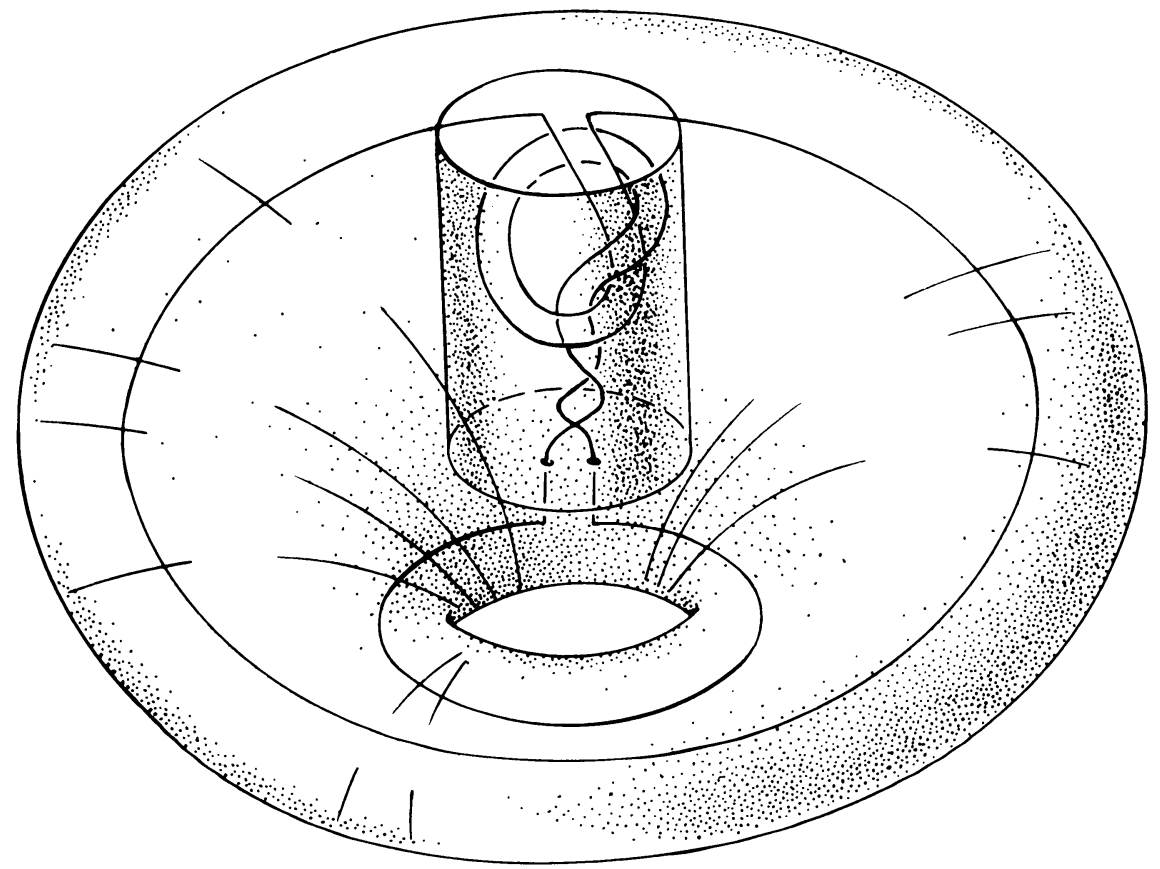

FIGURE 1 


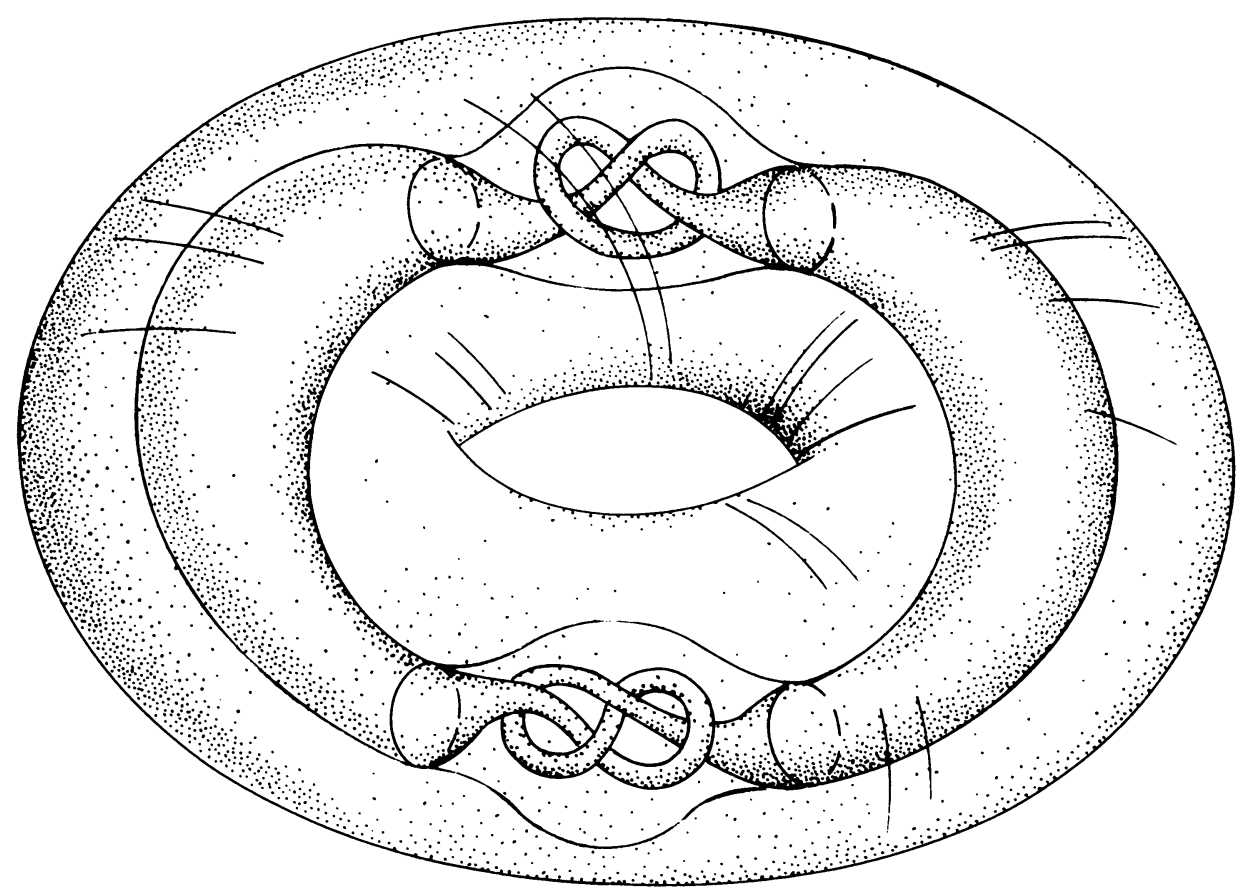

FIGURE 2

$B$ ties a figure eight in $A$, and $C$ ties a trefoil in $B$; and $A \supset B^{\prime} \supset C$ where $B^{\prime}$ ties a trefoil in $A$, and $C$ ties a figure eight in $B^{\prime}$.

For our last example we need:

Lemma 4.9. Suppose $(A, B)$ is a twisted pair with $N(A, B)=k, k \geq 3$. If $f, g:(A, B) \rightarrow S^{3}$ are unknotted embeddings, then $g f^{-1}$ extends to a homeomorphism of $S^{3}$ to itself.

Proof. The lemma is equivalent to this: If $B \subset A \subset S^{3}$ are unknotted with $(A, B)$ a twisted pair having $N(A, B) \geq 3$, and $h: A \rightarrow A$ is a homeomorphism which does not extend to $S^{3}$, then $h(B)$ is knotted in $S^{3}$. Extendability depends only on the isotopy class of $h$. Any element of the mapping class group of $A$ (i.e., isotopy classes of homeomorphisms) is represented by a composition of reflections, which extend to $S^{3}$, with $d^{n}$. Here $d$ is the homeomorphism which, on $S^{1} \times D^{2}$, sends $(\psi,(r, \mu))$ to $(\psi,(r, \mu+\psi))($ a Dehn twist). Thus we need only show that $d^{n}(B)$ is knotted in $S^{3}$ for $n \neq 0$. If $(m, l)$ is a meridian-longitude pair for $A$, then, on $\partial A, d^{n}(m) \sim m$, while $d^{n}(l) \sim l+n * m$. A core of $B$ is parallel to $b \subset \partial A$, where $b \sim k * l \pm m$. Then $d^{n}(b) \sim k * d^{n}(l) \pm d^{n}(m)=k * l+(n * k \pm 1) * m$. This is knotted unless $|n * k \pm 1|=1$, which is false since $k \geq 3$ and $n \neq 0$.

For our example, let $A \supset B \supset C$ be solid tori in $S^{3}$ so that $(A, B)$ and $(B, C)$ are the same twisted pair with $N(A, B) \geq 3$; so that $A$ and $B$ are unknotted in $S^{3}$, but $(B, C)$ is embedded with a single Dehn twist. Now $C$ is knotted in $S^{3}$, and we claim that if $f: A \rightarrow S^{3}$ is any unknotted embedding, then $f(C)$ is knotted in $S^{3}$. Suppose, to the contrary, that $f: A \rightarrow S^{3}$ is an embedding with both $A^{\prime}=f(A)$ and $C^{\prime}=f(C)$ unknotted. By Theorem 1.3, $B^{\prime}=f(B)$ is also unknotted. By Lemma 4.9, $f^{-1}$ extends to $S^{3}$ which 
contradicts the fact that $C=f^{-1}\left(C^{\prime}\right)$ is knotted. Using the construction in $\S 5$ of [10], make a genus one Whitehead manifold $O$ with a prime sequence $\left\{V_{n}\right\}$ so that $\left(V_{3 n+3}, V_{3 n+2}\right)$ is Whitehead's pair, while $V_{3 n+2} \supset V_{3 n+1} \supset V_{3 n}$ is homeomorphic to $A \supset B \supset C$. Then $\left(V_{n+1}, V_{n}\right)$ is unknotted for every $n$, but $\left(V_{3 n+2}, V_{3 n}\right)$ is knotted for every $n$. It follows that $O$ cannot be embedded in a compact 3-manifold.

A question, related to the above, that we have not been able to resolve is this: If $\left\{V_{n}\right\}$ is a prime sequence for an irreducible genus one Whitehead manifold $O$ which embeds in a compact 3-manifold, is the homeomorphism type of $O$ determined by the homeomorphism types of the pairs $\left(V_{n+1}, V_{n}\right)$ ?

\section{REFERENCES}

1. R. H. Bing, Necessary and sufficient conditions that a 3-manifold be $S^{3}$, Ann. of Math. 68 (1958), 17-37.

2. E. M. Brown, Contractible 3-manifolds of finite genus at infinity, Trans. Amer. Math. Soc. 245 (1978), 503-514.

3. R. H. Crowell and R. H. Fox, Introduction to knot theory, Graduate Texts in Math., vol. 57, Springer-Verlag, New York, 1963.

4. J. Glimm, Two cartesian products which are euclidean spaces, Bull. Soc. Math. France $\mathbf{8 8}$ (1960), 131-135.

5. W. Haken, Some results on surfaces in 3-manifolds, Studies in Modern Topology, Math. Assoc. Amer., Prentice-Hall, Englewood Cliffs, N.J., 1968, pp. 39-98.

6. J. Hemple, 3-manifolds, Ann. of Math. Stud., No. 86, Princeton Univ. Press, Princeton, N.J., 1976.

7. W. Jaco, Lectures on three-manifold topology, CBMS Regional Conf. Ser. in Math., no. 43, Amer. Math. Soc., Providence, R.I., 1980.

8. J. M. Kister and D. R. McMillan, Jr., Locally Euclidian factors of $E^{4}$ which cannot be embedded in $E^{3}$, Ann. of Math. 76 (1962), 541-546.

9. W. Magnus, A. Karrass, and D. Solitar, Combinatorial group theory, Interscience, New York, 1966.

10. D. R. McMillan, Jr., Some contractible open three-manifolds, Trans. Amer. Math. Soc. 102 (1962), 373-382.

11. D. R. McMillan, Jr. and T. L. Thickston, Open three-manifolds and the Poincaré conjecture, Topology 19 (1980), 313-320.

12. D. R. McMillan, Jr. and E. C. Zeeman, On contractible open manifolds, Proc. Cambridge Philos. Soc. 58 (1962), 221-224.

13. R. Myers, Contractible open 3-manifolds which are not covering spaces, Topology 27 (1988), 27-35.

14. H. Neumann, Generalized free products with amalgamated subgroups, Amer. J. Math. 70 (1948), 590-625.

15. V. Poénau, A remark on simply-connected 3-manifolds, Bull. Amer. Math. Soc. 80 (1974), 1203-1204.

16. H. Schubert, Knoten und Vollringe, Acta Math. 90 (1953), 131-286.

17. J. Stallings, On the loop theorem, Ann. of Math. 72 (1960), 12-19.

18. J. H. C. Whitehead, A certain region in Euclidean 3-space, Proc. Nat. Acad. Sci. U.S.A. 21 (1935), 364-366.

19. __ A certain open manifold whose group is unity, Quart. J. Math. 6 (1935), 268-279.

Department of Mathematics and Computer Science, Dartmouth College, Hanover, NEW HAMPSHIRE 03755 